\title{
Left ventricular noncompaction (LVNC) and low mitochondrial membrane potential are specific for Barth syndrome
}

\author{
Agnieszka Karkucinska-Wieckowska - Joanna Trubicka - Bozena Werner • \\ Katarzyna Kokoszynska • Magdalena Pajdowska • Maciej Pronicki • \\ Elzbieta Czarnowska • Magdalena Lebiedzinska • Jolanta Sykut-Cegielska • \\ Lidia Ziolkowska • Weronika Jaron • Anna Dobrzanska • Elzbieta Ciara • \\ Mariusz R. Wieckowski • Ewa Pronicka
}

Received: 13 July 2012 /Revised: 28 December 2012 / Accepted: 2 January 2013 / Published online: 30 January 2013

(C) The Author(s) 2013. This article is published with open access at Springerlink.com

\begin{abstract}
Barth syndrome (BTHS) is an X-linked mitochondrial defect characterised by dilated cardiomyopathy, neutropaenia and 3-methylglutaconic aciduria (3-MGCA). We report on two affected brothers with c.646G $>$ A (p.G216R) $T A Z$ gene mutations. The pathogenicity of the mutation, as indicated by the structure-based functional analyses, was further confirmed by abnormal monolysocardiolipin/cardiolipin ratio in dry blood spots of the patients as well as the
\end{abstract}

Communicated by: Eva Morava

Agnieszka Karkucinska-Wieckowska and Joanna Trubicka contributed equally to this work.

Electronic supplementary material The online version of this article (doi:10.1007/s10545-013-9584-4) contains supplementary material, which is available to authorised users.

\footnotetext{
A. Karkucinska-Wieckowska · M. Pronicki - E. Czarnowska Department of Pathology, The Children's Memorial Health Institute, Warsaw, Poland

J. Trubicka $\cdot$ E. Ciara

Department of Medical Genetics, The Children's Memorial Health Institute, Warsaw, Poland

B. Werner

Department of Pediatric Cardiology and General Pediatrics,

Medical University of Warsaw, Warsaw, Poland

K. Kokoszynska

Laboratory of Bioinformatics and Biostatistics, Maria

Sklodowska-Curie Memorial Cancer Center

and Institute of Oncology, Roentgena 5,

02-781 Warsaw, Poland

M. Pajdowska

Department of Biochemistry and Experimental Medicine,

Children's Memorial Health Institute, Warsaw, Poland
}

occurrence of this mutation in another reported BTHS proband. In both brothers, 2D-echocardiography revealed some features of left ventricular noncompaction (LVNC) despite marked differences in the course of the disease; the eldest child presented with isolated cardiomyopathy from late infancy, whereas the youngest showed severe lactic acidosis without 3-MGCA during the neonatal period. An examination of the patients' fibroblast cultures revealed that extremely low
M. Lebiedzinska $\cdot$ M. R. Wieckowski

Nencki Institute of Experimental Biology, Warsaw, Poland

J. Sykut-Cegielska $\cdot$ E. Pronicka $(\square)$

Department of Metabolic Diseases, The Children's Memorial

Health Institute, Aleja Dzieci Polskich 20,

04-730 Warsaw, Poland

e-mail: e.pronicka@czd.pl

L. Ziolkowska

Department of Cardiology, The Children's Memorial Health Institute, Warsaw, Poland

W. Jaron

Department of Surgery and Transplantation, The Children's Memorial Health Institute, Warsaw, Poland

A. Dobrzanska

Department of Neonatology, Pathology and Intensive Care,

The Children's Memorial Health Institute,

Warsaw, Poland 
mitochondrial membrane potentials (mt $\Delta \Psi$ about $50 \%$ of the control value) dominated other unspecific mitochondrial changes detected (respiratory chain dysfunction, abnormal ROS production and depressed antioxidant defense). 1) Our studies confirm generalised mitochondrial dysfunction in the skeletal muscle and the fibroblasts of BTHS patients, especially a severe impairment in the $\mathrm{mt} \Delta \Psi$ and the inhibition of complex $\mathrm{V}$ activity. It can be hypothesised that impaired $\mathrm{mt} \Delta \Psi$ and mitochondrial ATP synthase activity may contribute to episodes of cardiac arrhythmia that occurred unexpectedly in BTHS patients. 2) Severe lactic acidosis without 3methylglutaconic aciduria in male neonates as well as an asymptomatic mild left ventricular noncompaction may characterise the ranges of natural history of Barth syndrome.

\section{Introduction}

Barth syndrome (BTHS, OMIM 302060) is one of a large group of "mitochondrial diseases" affecting boys (Barth et al 1983; Barth et al 2004). The disorder is a rare inborn error of metabolism. Although hundreds of cases have been identified worldwide, the Human Gene Mutation Database (HGMD) includes only 54 patients for whom a genotype has been established.

BTHS is caused by mutations in the tafazzin gene (TAZ) and is inherited as an X-linked recessive trait (Barth et al 2004). Boys who are affected present with cardiopathy (and myopathy), neutropaenia and 3-methylglutaconic aciduria (3-MGCA) (Besley et al 1995; Chitayat et al 1992; Christodoulou et al 1994).

The cardiac involvement in BTHS may include: left ventricular (LV) dilation, hypertrophy and noncompaction with varying degrees of congestive heart failure as well as endocardial fibroelastosis. Although most patients have dilated cardiomyopathy (DCM) the prominent LV trabeculations in half of the cases suggest a form of noncompaction cardiomyopathy (NCCM), named also a left ventricular noncompaction (LVNC), and some have hypertrophic cardiomyopathy (HCM). Cardiac transplantation may be required in some BTHS patients (Spencer et al 2006; Hanke et al 2012).

BTHS is the first identified genetic defect from a rapidly expanding group of phospholipids defects (Lamari et al 2012), that directly affects cardiolipin (Houtkooper et al 2009a; b). Tafazzin is a component of the mitochondrial membrane that is necessary for the proper functioning of the electron transport chain (Brandner et al 2005; Claypool 2009; Haines2009).

Here, we present first Polish male siblings with BTHS associated with a recurrent $T A Z$ gene mutation and relatively mild course of the disease, in which the studies of mitochondrial function directly confirmed severe impairment in mitochondrial membrane potential $(\mathrm{mt} \Delta \Psi)$ and the inhibition of complex V activity.

\section{Materials}

This investigation conforms to the principles outlined in the Declaration of Helsinki, and the study protocol was approved by The Bioethical Commission of The Children's Memorial Health Institute. Two patients with BTHS and seven fibroblast cultures of patients with SCO2 (Pronicki et al 2010) and SURF1 (Pronicki et al 2008) mutations were studied.

\section{Patient 1}

The boy was born at full term from a second pregnancy (there was a maternal history of a single miscarriage). His birth weight was $3.090 \mathrm{~g}$, and his Apgar score after $5 \mathrm{~min}$ was a 10. During infancy, feeding difficulties, growth retardation (height 10th, weight corrected for height $<3$ rd percentile) and delayed motor development were observed (he sat at $8 \mathrm{mo}$, stood at $13 \mathrm{mo}$ and started to walk at $24 \mathrm{mo}$ ).

At the age of 14 months, after a respiratory infection, a cardiomyopathy occurred with severe heart failure (class IV according to modified Ross Heart Failure classification). After the treatment the patient's cardiac function progressively improved. Since the age of two years no symptoms or signs of heart failure have been found. ECG was without signs of left ventricular hypertrophy. Slight cardiomegaly was observed in roentgenogram. In echocardiography the dimensions of left atrium and left ventricle diminished, the diameter of left atrium was within normal limits and the left ventricular diameter was on the upper border. The left ventricular contractility markedly improved, SF was $30 \%$, EF $58 \%$ (modified Simpson formula), and the moderate mitral regurgitation was recorded. The ejection fraction reached about $64 \%$ at the age of $3.5-4$ years and the treatment with digoxin was stopped. In the next six following months further reduction of the left ventricular dimensions was observed and spironolacton was withdrawn.

Physical development has been delayed, always with weight below the 3 rd percentile.

Now, at the age of 9 , the patient is asymptomatic, treated only with captopril, and systematically followed by paediatric cardiologists and metabolic paediatricians.

\section{Patient 2}

The younger brother of patient 1 (no data from the fetal period is available) was healthy at birth (10 Apgar score after $5 \mathrm{~min}$, weight $3.240 \mathrm{~g}$ ), but he developed hypotonia, tremor and generalised oedema on the first day of life. Biochemical data showed the following: severe metabolic acidosis $(\mathrm{pH} 7.0$, 
$\left.\mathrm{HCO}_{3} 5.0 \mathrm{mmol} / \mathrm{L}\right)$, markedly increased lactate in the plasma $(23.1 \mathrm{mmol} / \mathrm{L})$ and urine, recurrent hypoglycaemia, rhabdomyolysis (CPK $7800 \mathrm{U} / \mathrm{L}$, control below $171 \mathrm{U} / \mathrm{L}$ ), liver failure and an abnormal aminoacidogram (hyperalaninaemia, hypertyrosinaemia and hypermetioninaemia). Generally the baby demonstrated "severe neonatal lactic acidosis". The structure of the brain and cerebellum was normal, as determined through MRI. The ECG after birth was in normal limits. Signs of right ventricular muscle hypertrophy were described in echocardiography performed soon after birth, which completely resolved within one month.

BTHS was suspected due to the positive family history, and pantothenate (a coenzyme A precursor) was administered as reported previously (Ostman-Smith et al 1994). Transient low serum biotinidase activity $(3.68 \mathrm{u} / \mathrm{mL}$, normal range $6-12$ $\mathrm{u} / \mathrm{mL}$ ) were observed, and biotin was supplemented. The patient's clinical condition and biochemical status systematically improved, and he was discharged without treatment at the age of 6 weeks. Recurrent skin Candida albicans infections were observed during the second year of life.

The boy never had any symptoms of heart failure therefore the regular cardiological assessment was not carried out. At age 3 years the boy is clinically asymptomatic. His weight and height are at the lower control limit (10th percentile).

\section{Methods}

Morphological and biochemical methods

Open muscle biopsy was performed at the age of 14 days in the younger brother (patient 2) and then in the older one (patient 1) at the age of 6 years. Histological, histochemical and spectrophotometric experiments on the muscle tissue were performed as described (Bancroft and Gamble 2001; Karczmarewicz et al 1997).

The muscle ultrastructure was evaluated under a JEM1010 electron microscope.

\section{Isolation of mitochondria from muscle biopsies}

Biopsy samples were stored at $-80{ }^{\circ} \mathrm{C}$ and then defrosted and cut into small pieces prior to resuspension in homogenisation buffer. Skeletal muscles were homogenised on ice. The homogenates were centrifuged twice at $1500 \mathrm{x} \mathrm{g}$ for $5 \mathrm{~min}$. The supernatant was collected and centrifuged at $10,000 \times \mathrm{g}$ for $10 \mathrm{~min}$. Finally, the mitochondrial pellets were frozen at $-80{ }^{\circ} \mathrm{C}$ until further use.

Blue native PAGE and in-gel activity assays

Blue native PAGE and an in-gel activity assay were performed with muscle biopsies, as described (Van Coster et al
2001). Briefly, the mitochondria that were isolated from the muscle biopsies were resuspended in aminocaproic acid buffer (1.5 M 6-aminocaproic acid and $50 \mathrm{mM}$ Bis-Tris, pH7.0) and supplemented with n-dodecyl-beta-D-maltoside at a final concentration of $1 \%$. The samples were incubated on ice for $20 \mathrm{~min}$ and then centrifuged at $20,000 \times \mathrm{g}$ for $15 \mathrm{~min}$. The protein concentrations of the supernatants containing the solubilised respiratory complexes were determined according to Bradford's method using the Bio-Rad protein estimation kit. The supernatant was supplemented with Serva Blue G (to a final concentration of $0.5 \%$ ) and subjected to native electrophoresis on an acrylamide gel gradient of 5-12\% in large format. To visualise the activity of the mitochondrial ATPase (complex V), the gel was incubated at $35{ }^{\circ} \mathrm{C}$ in a solution containing $35 \mathrm{mM}$ Tris- $\mathrm{HCl}, 270 \mathrm{mM}$ glycine, $14 \mathrm{mM}$ $\mathrm{MgSO}_{4}, 0.2 \% \mathrm{~Pb}\left(\mathrm{NO}_{3}\right)_{2}$ and $8 \mathrm{mM}$ ATP (pH7.8). To visualise complex I activity, the gel was incubated at $35^{\circ} \mathrm{C}$ in $3 \mathrm{mM}$ Tris- $\mathrm{HCl}$ (pH7.4) containing $60 \mu \mathrm{M}$ NADH and $245 \mu \mathrm{M}$ nitrotetrazolium blue (NBT). To visualise complex II activity, the gel was incubated at $35^{\circ} \mathrm{C}$ in $5 \mathrm{mM}$ phosphate buffer (pH7.4), $5 \mathrm{mM}$ EDTA, $10 \mathrm{mM} \mathrm{KCN}$, $50 \mathrm{mM}$ succinate, $0.2 \mathrm{mM}$ phenazine methosulphate and $245 \mu \mathrm{M}$ NBT. To visualise complex IV activity, the gel was incubated at $35{ }^{\circ} \mathrm{C}$ in $9 \mathrm{ml} 50 \mathrm{mM}$ phosphate buffer (pH7.4) supplemented with $5 \mathrm{mg}$ 3,3'-diamidobenzidine tetrahydrochloride (DAB), $200 \mu \mathrm{g}$ catalase, $10 \mathrm{mg}$ cytochrome $\mathrm{c}$ and $750 \mathrm{mg}$ sucrose.

Western blot analysis of the mitochondrial respiratory chain components

The levels of the individual subunits of the mitochondrial respiratory chain were assessed using the Total OXPHOS Human WB Antibody Cocktail (MitoSciences). SDS lysates of the muscle biopsies (20 $\mu \mathrm{g} / \mathrm{line})$ were electrophoretically separated with a $10 \%$ SDS-polyacrylamide gel (BioRad). The proteins were transferred onto a PVDF membrane using standard procedures. The membranes were blocked with $2 \%$ non-fat milk in TBS buffer containing $0.01 \%$ Tween 20 (Sigma Aldrich) for $1 \mathrm{~h}$. The membranes were immunoblotted with antibodies against the subunits of the individual respiratory chain complexes (1:5,000), followed by secondary HRP-conjugated antibodies $(1: 5,000)$ (Santa Cruz).

Measurement of the mitochondrial membrane potential $(\mathrm{mt} \Delta \Psi)$

The mitochondrial membrane potential ( $\mathrm{mt} \Delta \Psi)$ was measured using fluorescent indicators, as previously described (Lebiedzinska et al 2010; Karkucinska-Wieckowska et al 2011). Fibroblasts were grown in 24 -well plates, washed 
twice with PBS and incubated with $10 \mu \mathrm{M} \mathrm{JC}-1$ in PBS containing $5 \mathrm{mM}$ glucose for $10 \mathrm{~min}$ at $37{ }^{\circ} \mathrm{C}$. The cells were then washed twice with PBS. Green and red fluorescence were measured at wavelengths of $485 \mathrm{~nm}$ excitation/520 nm emission and $535 \mathrm{~nm}$ excitation/635 nm emission, respectively, using a microplate reader (Infinite M200, Tecan, Austria).

The data obtained from the Tecan microplate reader were calculated using Microsoft ${ }^{\mathrm{TM}}$ Excel 2005, and significance was assessed with Student's t-test.

Molecular analysis

Genomic DNA was extracted from Guthrie cards through incubation in the polymerase chain reaction (PCR) mixture at $95{ }^{\circ} \mathrm{C}$ for $50 \mathrm{~min}$. All of the exons of the $T A Z$ gene, including the flanking introns, were amplified with PCR using seven intron-specific primer pairs. Detailed PCR protocols and primer sequence information are available upon request. Direct sequencing of the PCR products was performed using a 3130 genetic analyser (Applied Biosystems, Foster City, CA, USA). The sequences were determined on both DNA strands from at least two independent PCR products. The analysed sequence fragments were compared with the TAZ cDNA (GenBank RefSeq: NM_000116.3) and protein (GenBank RefSeq: NP_000107.1) sequences using Mutation Surveyor software version 3.30 (Softgenetics, PA). The position of the identified nucleotide change was determined based on comparison with the reference sequence, with the A of the ATG translation initiation codon designated as nucleotide +1 .

\section{Structure-based functional analyses}

Sequences that were homologous to the human TAZ protein (NP_000107.1) were collected from the National Centre for Biotechnology Information (NCBI) RefSeq database. Selected sequences were aligned using the ClustalW algorithm. Globular regions were predicted using GlobPlot. Transmembrane domains were determined using TMHMM. The protein sequences were submitted to the Meta-BASIC and Protein Structure Prediction Meta-Server, which combines fold recognition and homology modelling methods. The secondary structure was determined using PsiPred. The obtained results were analysed with 3D-Jury, a consensus fold recognition method. Based on these results, the structural templates were mapped to the multiple sequence alignment of the TAZ homologs. Homology models for each of the individual domains were reconstructed using Modeller 9. The models were visualised using PyMOL and Discovery Studio Visualiser.

\section{Results}

Molecular study

The sequencing of the TAZ gene revealed a singlenucleotide mutation c. $646 \mathrm{G}>\mathrm{A}$ leading to the missense

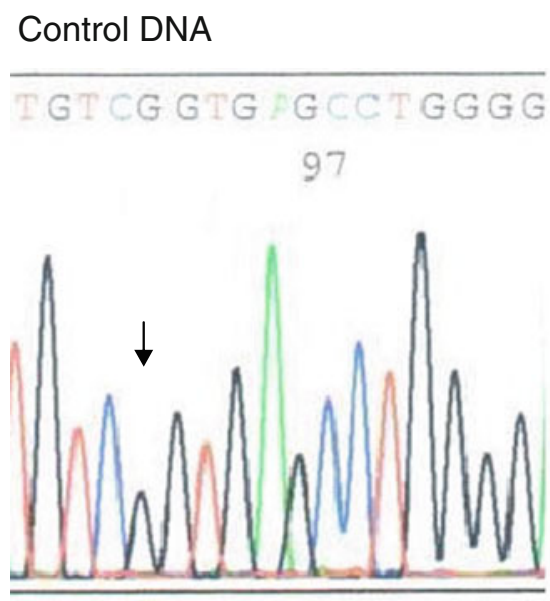

\section{Proband DNA}

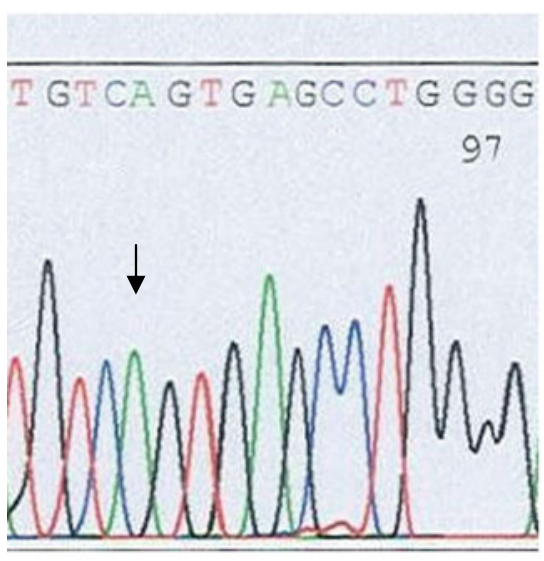

Mother DNA

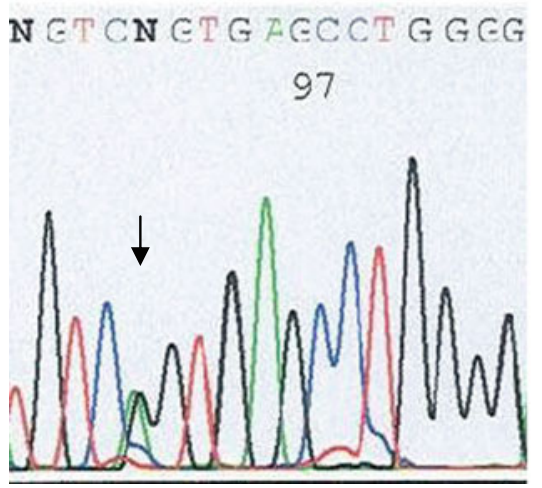

Fig. 1 The DNA sequence of TAZ exon 8 with the c.646G $>$ A mutation in the proband (in the hemizygous stage), the proband's mother (in the heterozygous stage) and a healthy control 
Fig. 2 Pedigree of the family
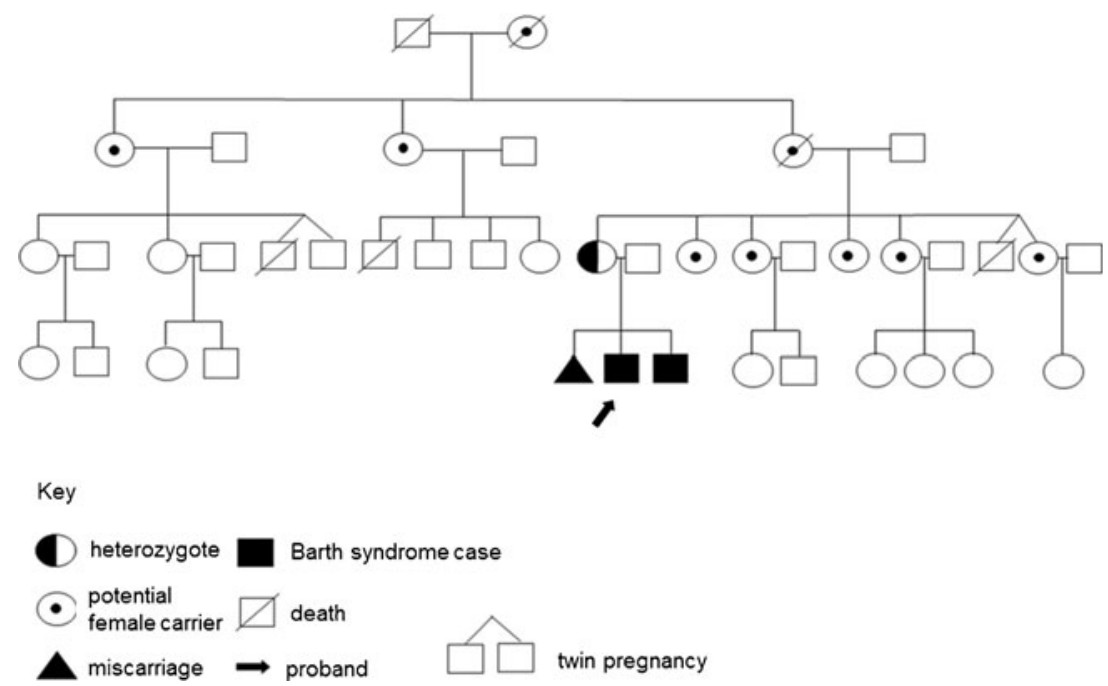

substitution p.Gly216Arg in the hemizygous state in both brothers and in the heterozygous state in their mother (Fig. 1). A pedigree analysis of the family (Fig. 2) revealed the deaths of at least three male relatives (late foetal, infantile and child deaths) and at least nine potential female carriers of the $T A Z$ mutation.

\section{Structure-based functional analyses}

The identified $T A Z$ gene mutation occurs in the splice site of exon 8 , which is highly conserved between species, suggesting that this variant may disrupt the function of the encoded protein (Supplementary Fig. 1). A detailed analysis of the position of the mutated Gly216 showed that this residue is facing the membrane, in which the N-terminal domain of the protein is anchored (Fig. 3). The Gly216 mutation segregating within this family occurs within a TAZ specific loop (position 216-232) that contains several conserved prolines that maintain specific loop flexibility.

\section{Biochemical abnormalities}

Excretion of 3-MGCA in the urine was increased in all of the measurements obtained, except for the first weeks of life of the younger patient.

Neutropaenia was not present at the onset. Neutrophils number fluctuated, and was normal in half of the measurements ranging from 375 to 3680 and from 230 to 2320 per $\mathrm{mm}^{3}$ (control above 1500 per $\mathrm{mm}^{3}$ ) respectively, in the older and younger brother. In the older patient, at the age of 4 years, an episode of severe neutropaenia was noted during the course of pneumonia.

Hypocholesterolaemia and hypocarnitinaemia were not evident The level of monolysocardiolipin/cardiolipin (MLCL/ CL) ratio measured at dry blood spots was increased in the older and younger brothers, respectively to 1.20 and 0.50 (normal value $0-0.30$ ). The analysis was performed in the Laboratory Genetic Metabolic Disease, Academic Medical Centre, Amsterdam.

\section{Cardiological study}

Multiple ECGs in patient 1 from the age of 14 months, showed ST-T abnormalities in the form of flattening and/or biphasic T (Supplementary Fig. 3). Similar biphasic T waves without signs of chamber hypertrophy were seen in patient 2 at the age of 3 years (Supplementary Fig. 4 and 5).

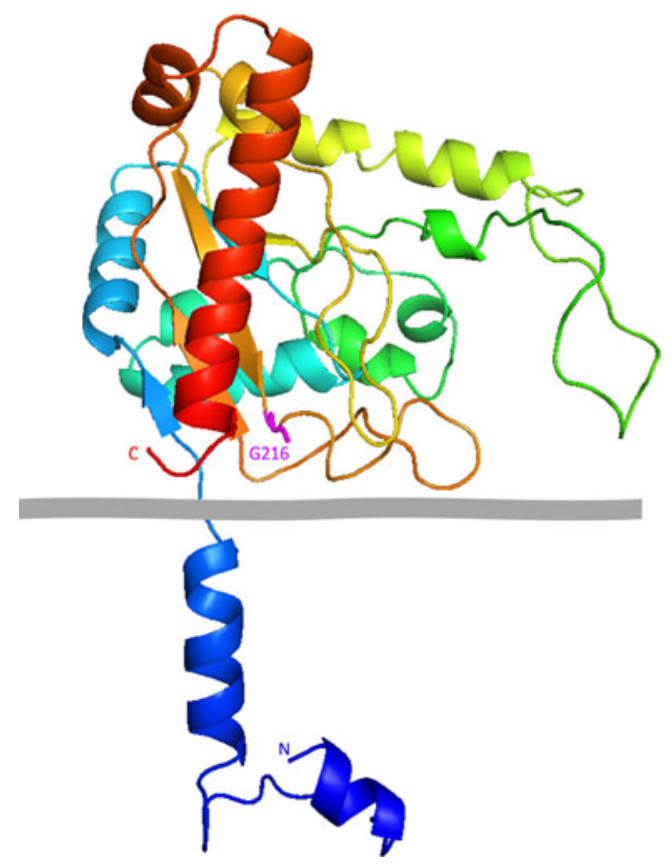

Fig. 3 The structural model of the TAZ protein rebuilt on PDB:1iuqA. The key G216 residue is indicated with a magenta stick. The dark blue element is an assumed transmembrane helix 
2D-echocardiography revealed some features of left ventricular noncompaction (LVNC) in both affected brothers. In patient 1 at the age of 8 years, two-layered myocardium was seen with prominent trabeculations in the apex, with noncompacted to compacted ratio about 1.6 (Supplementary Fig. 6).

In patient 2 , at the age of 3 years, two-layered myocardium was seen in systole with four trabeculations in the apical region, and the noncompacted to compacted ratio about 1.0.

Holter monitoring (multiple) showed normal sinus rhythm, normal conduction intervals, without evidence of arrhythmia in all recordings in both affected brothers.

\section{Mitochondrial study}

In a study on BTHS fibroblasts, the most evident abnormality was the impairment of mitochondrial membrane potential, $\mathrm{mt} \Delta \Psi$. Interestingly, the $\mathrm{mt} \Delta \Psi$ reduction was nearly identical in the fibroblasts of both affected brothers (40-50\% of control), and this reduction was more extreme than the reduction observed in the fibroblasts of patients with two other mitochondrial disorders ( $\mathrm{SCO} 2$ gene or $S U R F 1$ gene mutations). Control fibroblasts were included as a reference (Fig. 4).

Other less specific changes in mitochondrial morphology and function were also observed in the studied BTHS patients. Muscle histochemistry in one of the patients revealed moderate lipid accumulation, low COX activity and a weak SDH reaction, which developed only after prolonged incubation time (1.5 and $3 \mathrm{~h}$ ). Relatively low activity of the respiratory chain complex IV and II+III were found by spectrophotometry of muscle homogenate. The muscle electron microscopy showed polymorphic mitochondria of variable size $\left(0.02-0.16 \mu \mathrm{m}^{2}\right)$, decreased distance between inner membrane and myelin-like structure in mitochondrial matrix, and abundant glycogen and lipid droplets in contact with the mitochondrion. The results of blue native electrophoresis and an in-gel activity assay revealed a

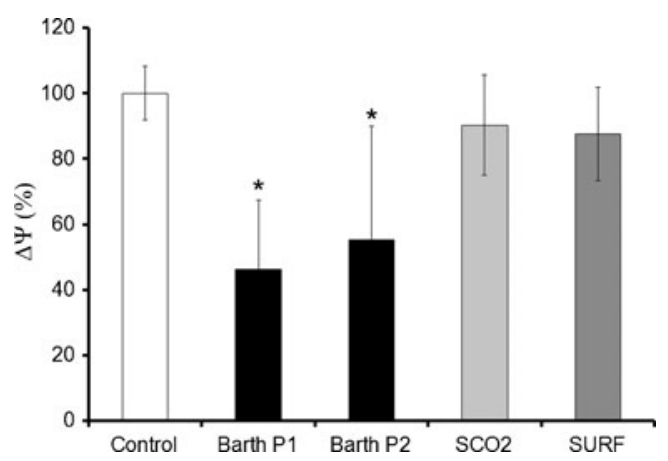

Fig. 4 The mitochondrial membrane potential in the fibroblasts of the two brothers with Barth syndrome ( 2 lines, $n=4)$, control fibroblasts ( 4 lines, $n=10$ ), SCO2-deficient fibroblasts ( 3 lines, $n=5)$ and SURF1deficient fibroblasts ( 4 lines, $n=6$ ). The data shown are the mean $\pm \mathrm{SD}$, ${ }^{*} p<0.05$ versus control decrease in the ATPase activity of complex V (Fig. 5). Western blot analysis (Fig. 6) revealed more complex mitochondrial dysfunction represented by lower complex V and I levels in the two studied patients versus control.

\section{Discussion}

Although most of the known $T A Z$ gene mutations are unique for probands and their families (Cantlay et al 1999; Bione et al 1996; Johnston et al 1997), the c.646G>A (p.G216R) mutation detected in this family has been previously described (Kuijpers et al 2004). The exchange of a small amino acid for a positively charged residue (Gly216 to Arg) in a region of TAZ specific loop is likely to influence the negatively charged cardiolipins that are localised to the mitochondrial membrane, resulting in failed cardiolipin biosynthesis and reduced ATP production. The pathogenicity of the p.G216R mutation, as indicated by the structure-based functional analyses, was further confirmed by observations of an abnormal MLCL/CL ratio in the described family.

The necessity of expanding the indications for differential diagnosis of BTHS for a pregnancy loss (as in the described family), and an idiopathic mitochondrial disease has been recently highlighted by Steward et al (2010). Prior experience with the first two Polish BTHS patients has shown that strict compliance with the requirements of the diagnostic triad for BTHS may delay a proper diagnosis (Sweeney et al 2008; Werner et al 2011). In the younger brother who had the initial diagnosis of "severe neonatal lactic acidosis", neither neutropaenia nor 3-MGCA was observed in the neonatal period, as reported (Schmidt et al 2004). After recovery from severe life-threatening neonatal disease, the boy did not show any cardiologic symptoms until the age of 3 years. Except for the positive family history and the presence of the $T A Z$ gene mutation, little laboratory evidence of BTHS existed. The symptoms of the older brother are more evident (stunted growth and cardiomyopathy) than in the younger brother but are still of only moderate intensity. Neutropaenia occurred only cyclically and was uncovered only when looked for on several occasions. The increased excretion of 3-MGCA was the most stable parameter of BTHS criteria triad in the described boys. However at neonatal period, a search for 3-MGCA in urine could be negative in affected patients. The increased excretion of 3-MGCA was not found in repeated GC-MS testing during the first three months of life of patient 2. Such phenomenon had been previously reported in one BTHS neonate (Schmidt et al 2004).

Clinical differences in the course of BTHS have been described in the literature, even for patients that bear identical mutations. Because no phenotypic association has been found among different types of mutations and any of the 


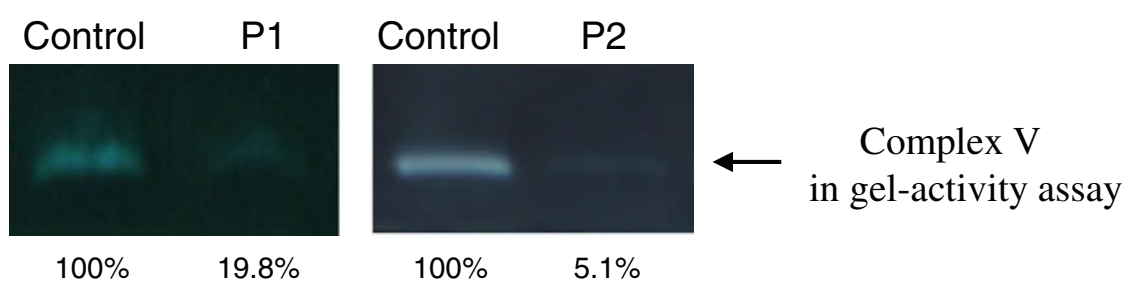

Fig. 5 Blue native electrophoresis and a complex V in-gel activity assay of the mitochondria isolated from the muscle biopsies of two brothers with Barth syndrome. A significant decrease in the activity of

clinical or laboratory abnormalities, it is likely that additional factors (acquired or inherited) modify the BTHS phenotype in individual affected patients, including members of the same family (Christodoulou et al 1994; Johnston et al 1997; Kelley et al 1991).

Isolated LVNC is frequently described in BTHS (Brandner et al 2005; Chen et al 2002). Furthermore, Spencer et al (2006) reported that many BTHS patients have qualitatively prominent trabeculations of the left ventricle, and two independent experts considered these patients to be in the left ventricular noncompaction group, even though they had not met the published criteria. Among the 30 patients with echocardiograms supporting this determination, only $16(53 \%)$ demonstrated an abnormal morphology of the left ventricle with increased trabeculations.

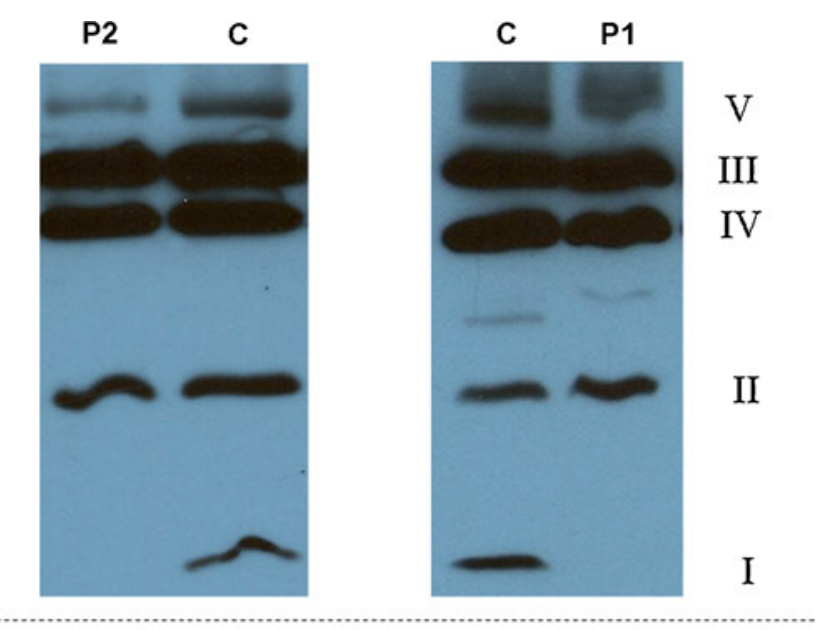

Longer exposure
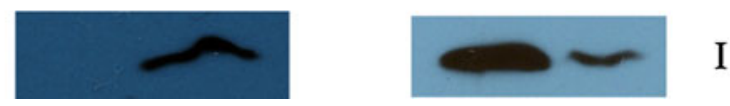

Fig. 6 A western blot analysis shows the mitochondrial respiratory chain composition in muscle biopsies of two brothers with Barth syndrome. A decreased amount of complex $\mathrm{V} \alpha$ subunit is observed compared with a healthy control. The $20 \mathrm{kDa}$ subunit of complex I was detectable in the older patient upon prolonged exposure. Densitometry analysis of the data is presented in Supplementary Fig. 2. P1: older brother; P2: younger brother complex V is observed compared with the control muscle biopsy. P1, older brother; P2, younger brother

LVNC represents a wide spectrum of morphological and clinical findings. 2D-echocardiography still is the method of choice in the diagnosis of this cardiomyopathy (Engberding et al 2007) and characteristic echocardiographic appearance may help to differentiate LVNC from other forms of cardiomyopathy like apical hypertrophic or dilated cardiomyopathy in patients with impaired left ventricular systolic function. However the echocardiographic criteria are still controversial. The fundamental feature of the condition is the presence of the two-layered myocardium: thin compact outer layer and thick noncompact endocardial layer with increased number of trabeculations and deep intertrabecular recesses communicating with left ventricular cavity imagined by colour Doppler. It is commonly known that twolayered myocardium can be sometimes demonstrated in healthy children and adults which can lead to misleading diagnosis of LVNC. Therefore, a quantification of the noncompacted to compacted ratio was implemented, for the first time by Chin et al (1990), then Ischida et al (1990), Jenni et al (2001) and others. There is no consensus in the literature if the ratio should be measured at end diastole or end systole neither of the cut-off value of it $(1.4 ; 2.0 ; 2.3)$. In our centre we perform the end-systolic measurement and for children we use the cut-off value 1.4 therefore the older brother fulfills the criteria of LVNC. The younger brother with the ratio about 1.0 meets the criteria recently proposed by Stollberger et al (2011). There is a broad discussion in the literature on the redefinition of the diagnostic criteria. The study of Saleeb et al (2012) demonstrated that the qualitative diagnosis and quantitative measurements are poorly reproducible between observers. Transesophageal echocardiography, 3D real time and contrast echocardiography play a complementary role in patients with poor quality of transthoracic images. The usefulness of cardiac magnetic resonance, computed tomography and invasive left ventriculography was documented in the small number of patients. All the mentioned methods could be helpful in the detailed diagnosis of LVNC as well as left ventricular function assessment and further studies on this issue are necessary.

In BTHS, repolarisation abnormalities predominantly include ST flattening or T-wave inversion (Ostman-Smith et al 1997; Spencer et al 2006). Ostman-Smith et al (1994) described 
the development of T-wave abnormalities in the form of a "camel's hump" biphasic T-wave and progressive T-wave inversion. These abnormalities were also seen in the presented BTHS brothers.

The data from the literature show that despite their currently reported good clinical condition, the boys in this study are at risk for ventricular tachycardia, which may lead to sudden cardiac death, and they require close cardiac monitoring (Barth et al 2004; Spencer et al 2005). The role of cardiolipin deficiency or altered distribution of the mitochondrial membrane phospholipids in the mechanism of arrhythmia has been considered (Spencer et al 2005).

We previously found that the decrease in $\mathrm{mt} \Delta \Psi$ was less pronounced in an infant with fatal mitochondrial cardiomyopathy (Lebiedzinska et al 2010) and a girl with a MEGDEL association (Karkucinska-Wieckowska et al 2011) (70\% and $75 \%$ of control, respectively). Moreover, BTHS lymphoblasts showed reduced $\mathrm{mt} \Delta \Psi$, as indicated with JC-1 probe measurements (Xu et al 2005). Similarly, decreased $m t \Delta \Psi$ and a lower activity of the individual respiratory chain complexes have also been reported by Besley et al (1995).

Tafazzin deficiency in BTHS leads to a cardiolipin remodelling defect (Houtkooper et al 2009a; Vrecken et al 2000) that affects the assembly and stability of supercomplexes of complex III and IV (and most likely also of complex V) in the inner mitochondrial membrane. These alterations result in the impairment of respiratory chain activity (Brandner et al 2005; Arnold et al 1998; Schagger and Pfeiffer 2000) and reduced ATP synthesis in BTHS (Haines 2009). Our studies show that reduced mitochondrial ATP synthase activity is accompanied by altered levels of its $\alpha$ subunit (Fig. 6). Moreover, a decrease in ATP synthase activity occurs not only with BTHS but also with other types of 3-MGCA (Besley et al 1995; Sperl et al 2006; Wortmann et al 2010).

Interestingly, we have previously observed increased ROS production, alterations in antioxidant defence and an increased level of protein carbonylation in BTHS fibroblasts (Lebiedzinska et al 2010). To our knowledge, these findings have not been previously reported. Thus, one may speculate that the natural course of the disease can be influenced, for example, by a background profile of antioxidant defence capacity in an individual patient.

\section{Summary}

1) Our studies confirm generalised mitochondrial dysfunction in the skeletal muscle and the fibroblasts of BTHS patients, especially a severe impairment in the mitochondrial membrane potential and the inhibition of complex V activity.
2) It can be hypothesised that impaired $\mathrm{mt} \Delta \Psi$ and mitochondrial ATP synthase activity may contribute to episodes of cardiac arrhythmia that occurred unexpectedly in BTHS patients (Brown and O'Rourke 2010; Spencer et al 2005).

3) Severe lactic acidosis without 3-methylglutaconic aciduria in male neonates as well as asymptomatic left ventricular noncompaction at ECHO record may characterise a range of natural history of Barth syndrome.

Acknowledgments This work was supported by the Polish Ministry of Science and Higher Education under the grants NN407 075137 and NN407 118939, the Polish Mitochondrial Network and the Internal Project of The Children's Memorial Health Institute No 117/09, No 119/09 and No 217/12. ML was the recipient of a fellowship from the Foundation for Polish Science (Program Start) and ML and JT an L'Oreal fellowship (for women in science).

Details of funding The authors confirm independence from the sponsors; the content of the article has not been influenced by the sponsors.

\section{Conflict of interest None.}

Open Access This article is distributed under the terms of the Creative Commons Attribution License which permits any use, distribution, and reproduction in any medium, provided the original author(s) and the source are credited.

\section{References}

Arnold I, Pfeiffer K et al (1998) Yeast mitochondrial F1F0-ATP synthase exist as a dimmer-specific subunits. EMBO J 17: $7170-7178$

Bancroft JD, Gamble M (2001) Theory and practice of histological techniques, 5th edn. Churchill Livingstone, Edinburgh

Barth PG, Scholte HR et al (1983) An X-linked mitochondrial disease affecting cardiac muscle, skeletal muscle and neutrophil leucocytes. J Neurol Sci 62:327-355

Barth PG, Valianpour F et al (2004) X-linked cardioskeletal myopathy and neutropenia (Barth syndrome): an update. Am J Med Genet 126A:349-354

Besley GTN, Lendon M et al (1995) Mitochondrial complex deficiencies in a male with cardiomyopathy and 3-methylglutaconic aciduria. J Inherit Metab Dis 18:221-223

Bione S, D'Adamo P et al (1996) A novel X-linked gene, G4.5. is responsible for Barth syndrome. Nature Genet 2:385-389

Brandner K, Mick DU et al (2005) Taz1 an outer mitochondrial membrane protein, affects stability and assembly of inner membrane protein complexes: implications for Barth syndrome. Mol Biol Cell 16:5201-5214

Brown DA, O'Rourke B (2010) Cardiac mitochondria and arrhythmias. Cardiovasc Res 88:241-248

Cantlay AM, Shokrollabi K et al (1999) Genetic analysis of the G4.5 gene in families with suspected Barth syndrome. J Pediat 135:311-315

Chen R, Tsuji T et al (2002) Mutation analysis of the G4.5 gene in patients with isolated left ventricular noncompaction. Mol Genet Metab 77:319-325 
Chin TK, Perloff JK, Wiliams RG, Jue K, Mohrmann R (1990) Isolated noncompaction of left ventricular myocardium: A study of eight cases. Circulation 82:507-513

Chitayat D, Chemke J et al (1992) 3-methylglutaconic aciduria; a marker for as yet unspecified disorders and the relevance of prenatal diagnosis in a 'new' type ('type 4'). J Inherit Metab Dis 15:204-212

Christodoulou J, McInnes RR et al (1994) Barth syndrome; clinical observations and genetic linkage studies. Am J Med Genet $50: 255-264$

Claypool SM (2009) Cardiolipin, a critical determinant of mitochondrial carrier protein assembly and function. Biochim Biophys Acta 1788:2059-2068

Engberding R, Yelbuz TM, Breithardt (2007) Isolated noncompaction of the left ventricular myocardium. A review of the literature two decades after the initial case description. Clin Res Cardiol 96:481-488

Haines TH (2009) A new look at cardiolipin. Bioch Biophys Acta 1788:1997-2002

Hanke SP, Gardner AB et al (2012) Left ventricular noncompaction cardiomyopathy in Barth syndrome: An example of an undulating cardiac phenotype necessitating mechanical circulatory support as a bridge to transplantation. Pediatr Cardiol. doi:10.1007/s00246012-0258-Z

Houtkooper RH, Rodenburg RJ (2009a) Cardiolipin and monosylocardiolipin analysis in fibroblasts, lymphocytes, and tissues using high-performance liquid chromatography-mass spectrometry as a diagnostic test for Barth syndrome. Analyt Biochem 387:230-237

Houtkooper RH, Turkenburg M (2009b) The enigmatic role of tafazzin in cardiolipin metabolism. Biochim Biophys Acta 1788:2003-2014

Ischida F, Hamamichi $Y$ et al (1990) Clinical features of isolated noncompaction of the ventricular myocardium: long term clinical course, hemodynamic properties, and genetic background. J Am Coll Cardiol 34:233-240

Jenni R, Oechslin E et al (2001) Echocardiographic and pathoanatomical characteristics of isolated left ventricular noncompaction: a step towards classification as a distinct cardiomyopathy. Heart 86:666671

Johnston J, Kelley RI et al (1997) Mutation characterization and genotype phenotype correlation in Barth syndrome. Am J Hum Genet 61:1053-1058

Karczmarewicz E, Bielecka L et al (1997) Analytical reliability of spectrophotometric analysis of the activity of mitochondrial respiratory chain complexes in muscle homogenates (in Polish). Diagn Lab 33:493-503

Karkucinska-Wieckowska A, Lebiedzinska M et al (2011) Increased reactive oxygen species (ROS) production and low catalase level in fibroblasts of a girl with MEGDEL association (Leigh syndrome, deafness, 3-methylglutaconic aciduria). Folia Neuropathol 49:56-63

Kelley RI, Clark BJ et al (1991) X-linked cardiomyopathy, neutropenia, growth retardation, and 3-methylglutaconic aciduria. J Pediat 119:738-745

Kuijpers TW, Maianski NA et al (2004) Neutrophils in Barth syndrome (BTHS) avidly bind annexin-V in the absence of apoptosis. Blood 103:3915-3923
Lamari F, Mochel F et al (2012) Disorders of phospholipids and fatty acids biosynthesis: toward a new category of inherited metabolic diseases. J Inherit Metab Dis doi:10.1007/s10545-012-9509-7

Lebiedzinska M, Karkucinska-Wieckowska A et al (2010) Oxidative stress-dependent p66Shc phosphorylation in skin fibroblasts of children with mitochondrial disorders. Biochim Biophys Acta Bioenergetics 1797:952-960

Ostman-Smith I, Brown G et al (1994) Dilated cardiomyopathy due to type II X-linked 3-methylglutaconic aciduria: successful treatment with pantothenic acid. Brit Heart J 73:349-353

Pronicki M, Matyja E et al (2008) Light and electron microscopy characteristics of the muscle of patients with SURF1 gene mutations associated with Leigh disease. J Clin Pathol 61:460-466

Pronicki M, Kowalski P et al (2010) A homozygous mutation in the $\mathrm{SCO} 2$ gene causes a spinal muscular atrophy like presentation with stridor and respiratory insufficiency. Eur J Paediatr Neurol 14:253-260

Saleeb SF, Margossian R et al (2012) Reproducibility of echocardiographic diagnosis of left ventricular noncompaction. J Am Soc Echocardiogr 25:194-202

Schagger H, Pfeiffer K (2000) Supercomplexes in the respiratory chains in yeast and mammal mitochondria. EMBO J 19:1777-1783

Schmidt MR, Birkebaek N et al (2004) Barth syndrome without 3methylglutaconic aciduria. Acta Paediatr 93:419-429

Spencer CT, Byrne BJ et al (2005) Ventricular arrhythmia in the $\mathrm{X}$ - linked cardiomyopathy Barth syndrome. Pediatr Cardiol 26:632-637

Spencer CT, Bryant RM et al (2006) Cardiac and clinical phenotype in Barth syndrome. Pediatrics 118:e337-e346

Sperl W, Jesina P et al (2006) Deficiency of mitochondrial synthase of nuclear genetic origin. Neuromuscul Dis 16:821-829

Steward CG, Newbury-Ecob RA et al (2010) Barth syndrome: an Xlinked cause of fetal cardiomyopathy and stillbirth. Prenat Diagn 30:970-976

Stollberger C, Gerecke B, Finsterer J, Engberding R (2011) Refinement of echocardiographic criteria for left ventricular noncompaction. Int J Cardiol. doi:10.1016/j.ijcard.2011.08.845

Sweeney RT, Davis GJ et al (2008) Cardiomyopathy of unknown etiology: Barth syndrome unrecognized. Congenit Heart Dis $3: 443-448$

Van Coster R, Smet J et al (2001) Blue native polyacrylamide gel electrophoresis: a powerful tool in diagnosis of oxidative phosphorylation defects. Pediatr Res 50:658-665

Vrecken P, Valianpour F et al (2000) Defective remodeling of cardiolipin and phosphatidylglycerol in Barth syndrome. Biochim Biophys Res Comun 27:378-382

Werner B, Trubicka J et al (2011) Clinical and diagnostic aspects of Barth syndrome (X-linked cardiomyopathy). Kardiologia Polska 69:1177-1180

Wortmann SB, Kluijtmans LA et al (2010) The 3-methylglutaconic acidurias: what's new? J Inherit Metab Dis doi:10.1007/s10545010-9210-7

$\mathrm{Xu}$ Y, Sutachan JJ et al (2005) Characterization of lymphoblast mitochondria from patients with Barth syndrome. Lab Invest $85: 823-830$ 